\title{
Synthetic promoters consisting of defined cis-acting elements link multiple signaling pathways to probenazole-inducible system ${ }^{* \#}$
}

\author{
Zheng ZHU ${ }^{1,3}$, Jiong $\mathrm{GAO}^{2}$, Jin-xiao YANG ${ }^{2}$, Xiao-yan $\mathrm{WANG}^{2}$, \\ Guo-dong REN ${ }^{2}$, Yu-long DING ${ }^{1,4}$, Ben-ke KUAI ${ }^{\dagger+1,2}$ \\ ( ${ }^{1}$ The Southern Modern Forestry Collaborative Innovation Center, Nanjing Forestry University, Nanjing 210037, China) \\ ( ${ }^{2}$ State Key Laboratory of Genetic Engineering and Institute of Plant Biology, School of Life Sciences, Fudan University, Shanghai 200438, China) \\ ( ${ }^{3}$ College of Biology and the Environment, Nanjing Forestry University, Nanjing 210037, China) \\ ( ${ }^{4}$ Bamboo Research Institute, Nanjing Forestry University, Nanjing 210037, China) \\ †E-mail: bkkuai@fudan.edu.cn \\ Received July 19, 2014; Revision accepted Nov. 6, 2014; Crosschecked Mar. 18, 2015
}

\begin{abstract}
Probenazole (3-allyloxy-1,2-benzisothiazole-1,1-dioxide, PBZ), the active component of Oryzemate, could induce systemic acquired resistance (SAR) in plants through the induction of salicylic acid (SA) biosynthesis. As a widely used chemical inducer, PBZ is a good prospect for establishing a new chemical-inducible system. We first designed artificially synthetic promoters with tandem copies of a single type of cis-element (SARE, JERE, GCC, GST1, HSRE, and W-box) that could mediate the expression of the $\beta$-glucuronidase (GUS) reporter gene in plants upon PBZ treatment. Then we combined different types of elements in order to improve inducibility in the PBZ-inducible system. On the other hand, we were surprised to find that the cis-elements, which are responsive to jasmonic acid (JA) and ethylene, also responded to PBZ, implying that SA, JA, and ethylene pathways also would play important roles in PBZ's action. Further analysis demonstrated that PBZ also induced early events of innate immunity via a signaling pathway in which $\mathrm{Ca}^{2+}$ influx and mitogen-activated protein kinase (MAPK) activity were involved. We constructed synthesized artificial promoters to establish a PBZ chemical-inducible system, and preliminarily explored SA, JA, ethylene, calcium, and MAPK signaling pathways via PBZ-inducible system, which could provide an insight for in-depth study.
\end{abstract}

Key words: Probenazole, Systemic acquired resistance (SAR), Cis-acting element, Chemical-inducible system, Synthetic promoter doi: 10.1631 jzus.B1400203

Document code: A

CLC number: Q789

\section{Introduction}

In eukaryotes, the promoter is the core DNA region that controls the initiation of gene expression. A

\footnotetext{
$\ddagger$ Corresponding author

* Project supported by the National Key Project of Transgenic Variety Development of China (Nos. 2011ZX08009-004 and 2013ZX08009004), Shanghai Key Laboratory of Bio-Energy Crops, and the Priority Academic Program Development of Jiangsu Higher Education Institutions (PAPD), China

${ }^{\#}$ Electronic supplementary materials: The online version of this article (http://dx.doi.org/10.1631/jzus.B1400203) contains supplementary materials, which are available to authorized users

(D) ORCID: Zheng ZHU, http://orcid.org/0000-0002-5379-9085; Ben-ke KUAI, http://orcid.org/0000-0003-0523-6381

(C) Zhejiang University and Springer-Verlag Berlin Heidelberg 2015
}

gene promoter usually contains a battery of cis-acting regulatory elements, with their combined effect shaping the expression profile in response to both developmental and environmental signals (Rushton et al., 2002). Promoters of plant hormone and several other key signaling pathway genes have been extensively studied, and a list of cis-acting elements has been isolated, which exhibit diversified responsive characteristics (Rushton and Somssich, 1998; Rushton et al., 2002). The GST1 box is one of the elements that were isolated early on from the promoter of the potato GST1 gene encoding a glutathione $S$-transferase, which is used as a reliable marker for the generation of reactive oxygen species (ROS) (Levine et al., 1994; 
Strittmatter et al., 1996); and SARE, a cis-acting element responsive to salicylic acid (SA), was then isolated from the upstream region of tobacco $P R 2$ gene (Shah and Klessig, 1996). Then JERE was reported to modulate the expressions of both elicitorand jasmonate-responsive genes (Menke et al., 1999); GCC box, an ethylene-responsive element, was identified in the promoters of both ethylene signaling pathway genes and pathogen-related genes (OhmeTakagi et al., 2000). More recently, S box, a GCC-like element, was shown to be responsible for the activation of fungal elicitors-triggered gene expression (Kirsch et al., 2000; Rushton et al., 2002); HSRE, an HSR203 responsive element, was located in the promoter region of $H S R 203 \mathrm{~J}$ to mediate the hypersensitive response (HR) in Nicotiana tabacum (Pontier et al., 2001).

Recently, advances in promoter construction technology allow us to specifically manipulate the transcriptional activities of target genes. Combining pathogen and/or chemical-responsive defined cis-acting elements with constitutive promoters to generate inducible synthetic promoters could not only provide a precise control of transgene expression but also modulate its expression in various contexts (Venter, 2007; Mehrotra et al., 2011). In addition, these inducible synthetic promoters can be introduced into plants as molecular markers, which may provide a powerful approach for studying plant-pathogen interactions and characterizing signaling pathways induced by various chemicals at the molecular level.

Probenazole (PBZ) is the active component of Oryzemate, an environment-friendly rice blast controlling agrochemical since 1975 (Watanabe, 1977; Sekizawa and Watanabe, 1981). Previous reports revealed that many defense-related genes, including $P B Z 1$ and RPR1, as well as accumulation of superoxide and hydroxyl unsaturated fatty acids, are induced by PBZ in rice (Midoh and Iwata, 1996; Sakamoto et al., 1999; Yasuda et al., 2008). Moreover, PBZ triggers the activation of multiple defenserelated enzymes, including 1-aminocyclopropane-1carboxylic acid (ACC) synthase, peroxidase, phenylalanine ammonia-lyase, and lipoxygenase in rice seedlings inoculated with the blast fungi Magnaporthe grisea (Sekizawa and Watanabe, 1981; Nikolaev and Aver'yanov, 1991; Midoh and Iwata, 1997; Sasaki et al., 2004). Research carried out on Arabidopsis and tobacco revealed that, among chemicals being identified as systemic acquired resistance (SAR) activators, PBZ is the only one that can initiate the SA/NPR1 (nonexpressor of PR genes 1)-mediated defense signaling pathway (Yoshioka et al., 2001; Nakashita et al., 2002; Yasuda et al., 2008). However, it is not known yet how PBZ contributes to SA accumulation. In addition, the possibility cannot be ruled out that PBZ induces other pathways as well. To address these issues, we constructed a series of synthetic promoters by combining a minimal cauliflower mosaic virus (CaMV) $35 \mathrm{~S}$ promoter with a range of aforementioned defined defense-related cis-acting elements, either separately or in combination, with the $\beta$-glucuronidase $(G U S)$ gene as a reporter. By taking an advantage of the efficient Arabidopsis stable expression system, we demonstrate that, in addition to the SA signaling pathway, both jasmonic acid (JA) and ethylene signaling pathways are also somehow involved in the PBZ-induced immune response. In addition, $\mathrm{HR}, \mathrm{Ca}^{2+}$ signal and kinase activity likely play roles in the upstream of signal transduction triggered by PBZ. Moreover, we illustrate that, by combining diverse PBZ inducible cis-acting elements into one synthetic promoter, promoter efficacy can be significantly improved.

\section{Materials and methods}

\subsection{Plant growth conditions}

All Arabidopsis plants were grown in a short day condition ( $9 \mathrm{~h} \mathrm{light} / 15 \mathrm{~h}$ dark $)$ in a growth chamber at $(21 \pm 2){ }^{\circ} \mathrm{C}$.

\subsection{Plasmid construction of synthetic promoters fused with $G U S$ gene}

Both sense and antisense strands of a minimal CaMV 35S promoter $(-46,+1)$ were synthesized with a PstI site at the $5^{\prime}$ end and an $N c o$ I site at the $3^{\prime}$ end. After annealing, the double-stranded DNA fragment containing the Pst $\mathrm{I} / \mathrm{NcoI}$ overhangs was subcloned into the PstI/NcoI-digested pCAMBIA1301 vector, resulting in pCAMBIA1301-1. Promoter constructs were produced in a similar way by annealing sense and antisense oligoes, which contained an $X b a \mathrm{I}$ site at the $5^{\prime}$ end and a PstI site at the 3 ' end. These synthetic promoters were introduced into the $X b a \mathrm{I}$ and $P s t \mathrm{I}$ sites 
of the binary vector pCAMBIA1301-1 (Fig. 1a).

\subsection{Arabidopsis transformation}

The resulting constructs expressing the GUS reporter gene under respective synthetic promoters were introduced into the Agrobacterium tumefaciens strain GV3101. Plant transformation was performed as previously described (Clough and Bent, 1998) and transgenic plants were identified on Murashige and Skoog (MS) agar medium supplemented with $40 \mu \mathrm{g} / \mathrm{ml}$ hygromycin. T3 homozygotes were used for the GUS activity assay.

\subsection{PBZ, salicylic acid, $\mathrm{La}^{3+}$, K252a, and apigenin treatments of plants}

Four-week-old transgenic Arabidopsis plants were treated with $0.5 \mathrm{mmol} / \mathrm{L} \mathrm{PBZ}$ or $1 \mathrm{mmol} / \mathrm{L} \mathrm{SA}$ by root drenching and/or leaf spraying. Transgenic lines containing $4 \times \mathrm{HSRE}$ were pretreated with $4 \mu \mathrm{mol} / \mathrm{L}$ $\mathrm{K} 252 \mathrm{a}, 0.4 \mathrm{mmol} / \mathrm{L} \mathrm{La}^{3+}$, and $0.25 \mathrm{mmol} / \mathrm{L}$ apigenin prior to PBZ treatment, and then leaves were taken and frozen in liquid nitrogen for analysis.

\subsection{RNA extraction and qRT-PCR analysis}

Total RNA was isolated from $100 \mathrm{mg}$ leaves by TRIzol (Invitrogen, Carlsbad, CA, USA). The extracted RNA was dissolved in $20 \mu 1$ diethyl pyrocarbonate (DEPC)-treated water. The SuperScript ${ }^{\mathbb{B}}$ FirstStrand Synthesis Kit (Invitrogen, Carlsbad, CA, USA) was used for reverse transcription. The generated complementary DNA (cDNA) was subsequently used as a template for the quantitative real-time polymerase chain reaction (qRT-PCR) analysis. The qRT-PCR was performed using the SYBR ${ }^{\circledR}$ Green I PCR Kit (Toyobo, Osaka, Japan) on a Bio-Rad iCycler according to the manufacturer's instructions (Bio-Rad, Hercules, CA, USA). AtACT2 was used as a reference gene.

\subsection{Analysis of GUS activity}

Measurement of GUS activity was conducted according to Jefferson et al. (1987). In brief, $0.1 \mathrm{~g}$ of frozen leaf samples were homogenized in $0.2 \mathrm{ml}$ of pre-cooled lysis buffer $(0.1 \mathrm{~mol} / \mathrm{L}$ sodium phosphate $\mathrm{pH} 7.5,10 \%$ glycerol, and $1 \mathrm{mmol} / \mathrm{L}$ ethylenediaminetetraacetic acid (EDTA)) and then centrifuged at $4000 \mathrm{~g}$ for $10 \mathrm{~min}$ at $4{ }^{\circ} \mathrm{C}$. The supernatant was transferred to a new tube and used for measuring GUS activity. GUS activity was calculated as described by Jefferson et al. (1987).

\subsection{Microarray analysis}

Arabidopsis plants overexpressing NPR1 were grown under a short day condition $(9 \mathrm{~h}$ light $/ 15 \mathrm{~h}$ dark $)$ for about four weeks. Total RNAs of PBZ-pretreated plants and control were extracted by the RNeasy Mini Kit (Qiagen) and repeated isopropanol precipitations. The extracted total RNA was subject to cDNA and complementary RNA (cRNA) syntheses with the Affymetrix GeneChip One-Cycle Target Labeling Kit. Microarray was performed in Shanghai Biotechnology Corporation (China) according to the standard Affymetrix manual. Differential gene expression was calculated by their relative expression of a PBZ-treated sample as compared with that of a mock sample.

\section{Results}

\subsection{Minimal 35S CaMV promoter does not respond to PBZ treatment}

In this study, a variant pCAMBIA1301 binary expression vector, harboring GUS gene driven by the minimal CaMV 35S promoter, was used as a basic cloning vector, and a series of hormone-inducible constructs was constructed by placing defined elements to the $5^{\prime}$ of the minimal CaMV $35 \mathrm{~S}$ promoter, as shown in Fig. 1a. To clarify whether the minimal $35 \mathrm{~S}$ promoter responds to $\mathrm{PBZ}$ treatment, the cloning vector harboring the minimal $35 \mathrm{~S}$ promoter as well as the original pCAMBIA1301 harboring the full-length $35 \mathrm{~S}$ promoter was introduced into Arabidopsis plants separately. By examining the GUS activity of T3 transgenic lines after $0.5 \mathrm{mmol} / \mathrm{L}$ PBZ application, we showed that neither the minimal CaMV 35S promoter nor the full-length CaMV $35 \mathrm{~S}$ promoter responded to PBZ treatment (Fig. 1b). Moreover, the transgenic plants harboring the $35 \mathrm{~S}$ minimal promoter showed only a trace level of background GUS activity. These findings indicate that the cloning vector, named as pCAMBIA1301-1, is an ideal basic vector for generating PBZ-inducible constructs for the assessment of the responsiveness of diverse defined cis-elements to PBZ treatment. 
(a)

(b)
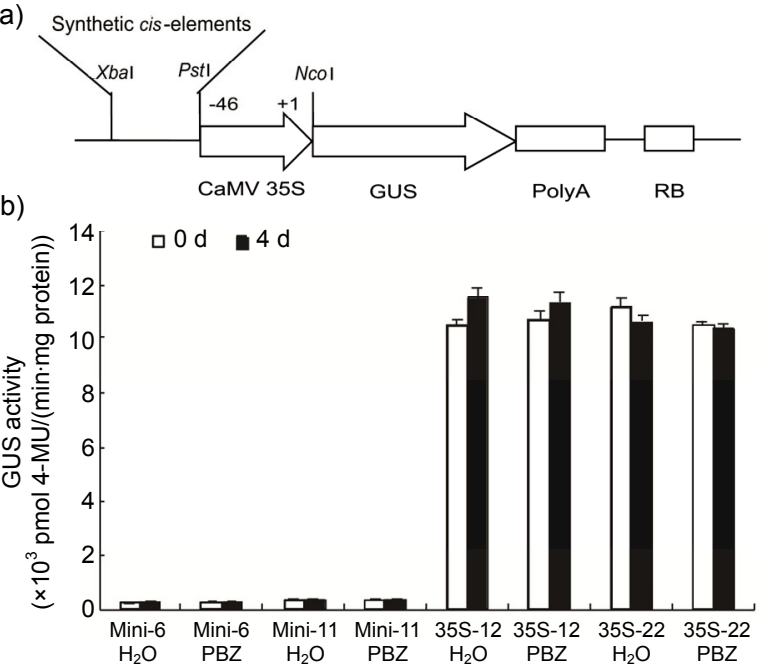

Fig. 1 Responsiveness of the $-46 \mathrm{CaMV} 35 \mathrm{~S}$ minimal promoter to $\mathrm{PBZ}$

(a) Scheme of the synthetic promoters. Elements were inserted between the $X b a \mathrm{I}$ and Pst I sites in 1301-1 upstream of the -46 CaMV $35 \mathrm{~S}$ minimal promoter. (b) GUS activity in the leaves of CaMV $35 \mathrm{~S}$ promoter transgenic T3 lines. Min-6 and Min-11 were two independent lines of the -46 CaMV 35S minimal promoter. 35S-12 and 35S-22 were two independent lines of the full-length CaMV 35S promoter. 4-MU: 4-methylumbelliferone. Error bars indicate standard deviations $(n=6)$

\subsection{SARE, an salicylic acid responsive element, responds to $\mathrm{PBZ}$ treatment}

Previous studies demonstrated that PBZ induces SAR through the enhancement of SA accumulation (Yoshioka et al., 2001; Yu et al., 2010b). To validate our PBZ-inducible system, we analyzed an SA responsive element, SARE, isolated from the upstream region of tobacco $P R 2$ gene in SA and PBZ treatments (Fig. 2a). Tandem repeats of four SAREs (4× SARE) were used to generate a synthetic promoter in order to improve its regulatory efficiency (Rushton et al., 2002). After introducing into Arabidopsis plants, the tandem repeats of four SAREs showed significant responses to both PBZ and SA (Fig. 2b). This indicates that our way of constructing synthetic promoters is valid and our system of assessing the responsiveness of these promoters is effective and efficient.

\subsection{JA and ethylene signaling pathways may be involved in the immune response triggered by PBZ}

To explore whether the JA and ethylene signaling pathways also play a role in the immune responses induced by PBZ, $4 \times$ JERE element (AGACCGCC) and $4 \times$ GCC box (AGCCGCC), responsive to jasmonate and ethylene respectively, were employed to construct two inducible constructs and their transgenic lines were then generated. Leaves of T3 transgenic lines were subject to GUS activity assay 0,1 , and $3 \mathrm{~d}$ after soil drenching treatment with $0.5 \mathrm{mmol} / \mathrm{L}$ PBZ. Both constructs were found to significantly respond to PBZ. The GUS activity in transgenic plants harboring $4 \times$ JERE increased continuously and reached a 9-fold increment by Day 3 after treatment (Fig. 3a). The GUS activity in the transgenic plants harboring $4 \times$ GCC increased by 4 -fold in a similar dynamic pattern $3 \mathrm{~d}$ after treatment (Fig. $3 b$ ). The highly inducible increments are partly attributable to their relatively low background expressions in the absence of PBZ. The fact that both the JA and ethylene signaling pathways respond to $\mathrm{PBZ}$ treatment suggests that, apart from SA signaling, both the JA signaling and ethylene signaling are also possibly involved in the immune response triggered by PBZ.

To further explore the involvement of JA and ethylene signaling pathways in the PBZ-induced system, we compared our microarray data on PBZ treatment with those published data on JA and ethylene treatment (Nemhauser et al., 2006). As listed in Tables S1 and S2, 94 genes were up-regulated by both methyl jasmonate (MeJA) and PBZ, and 27 genes were induced by both ACC and PBZ (Fig. 4a). In addition, qRT-PCR analysis further revealed that PBZ can induce the immune response by stimulating the expression of PDF1.2 (Fig. 4b), a gene that is specifically induced by JA and ethylene in Col-0 (Penninckx et al., 1996).

(a) SARE: AGTATAGGGGCAGCTTCGACCTCCTTCTCCGAA

(b)

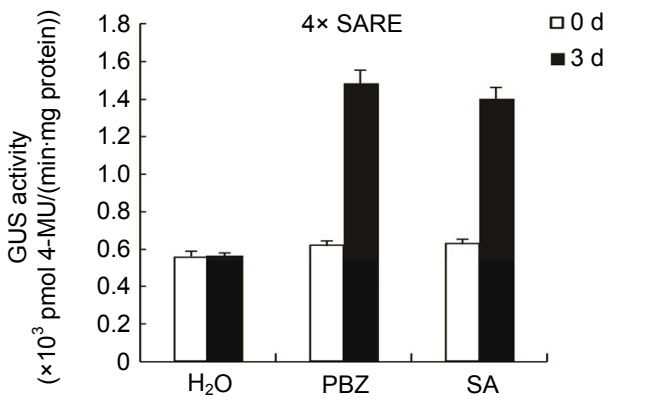

Fig. 2 PBZ-inducible promoter containing SARE elements (a) Sequence of the SARE element. Core sequences are shown in boldface. (b) GUS activity in the leaves of $4 \times$ SARE transgenic T3 lines. 4-MU: 4-methylumbelliferone. Error bars indicate standard deviations $(n=6)$ 
(a)

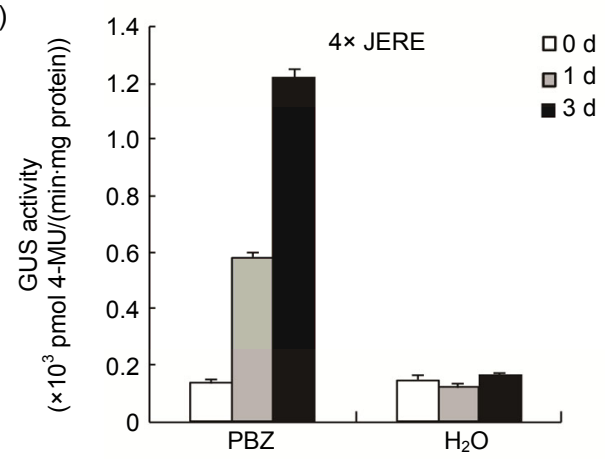

(b)

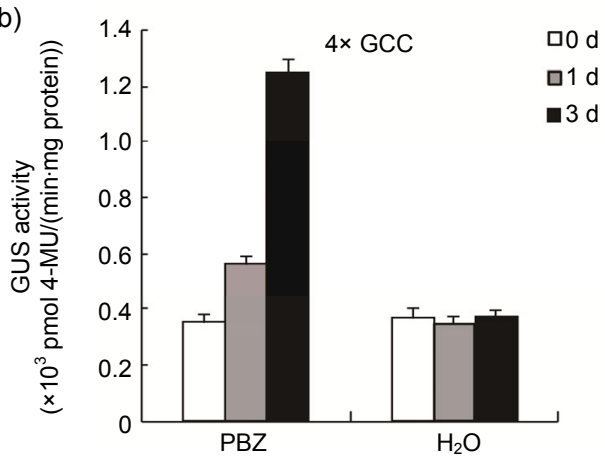

Fig. 3 PBZ inducibility of JERE and GCC boxes

(a) GUS activity in the leaves of $4 \times$ JERE transgenic T3 lines. (b) GUS activity in the leaves of $4 \times$ GCC transgenic T3 lines. 4-MU: 4-methylumbelliferone. Error bars indicate standard deviations $(n=6)$

(a)

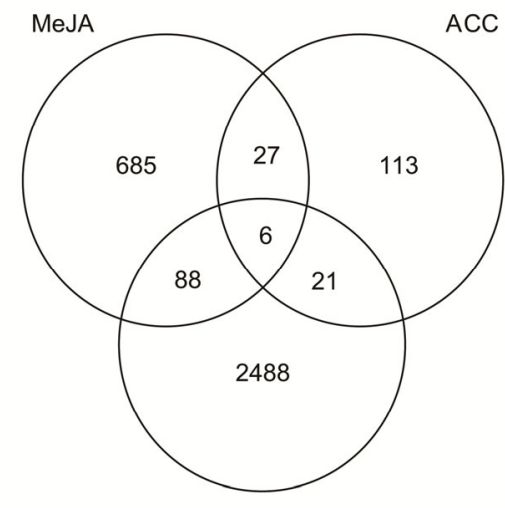

(b)

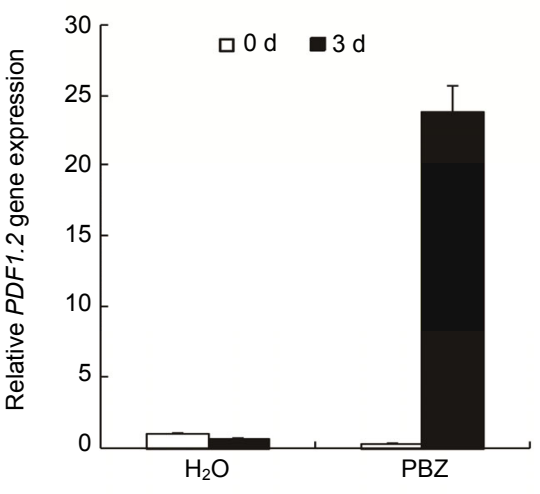

Fig. 4 Overlapping induction of gene expression by PBZ, JA, and ethylene

(a) Venn diagrams showing the numbers of genes induced by PBZ, MeJA, and ACC, respectively. Details about the shared up-regulated genes were listed in Tables S1 and S2. (b) The induction of PDF1.2 expression in Col-0 upon PBZ treatment. The transcript levels were quantified by qRT-PCR with ACTIN2 (ACT2) as an internal control. Error bars indicate standard deviations $(n=4)$

\subsection{Hypersensitive response is involved in the immune response triggered by $\mathrm{PBZ}$}

HR is an early event that contributes to plant resistance by restricting invading pathogens at the infection site, which may play an important role in stimulating a systemic signal for SAR (Heath, 2000). We therefore hypothesized that PBZ might stimulate the immune response via HR. To verify this, $4 \times$ HSRE and $4 \times$ GST1, which are associated with HR, were employed to construct inducible constructs (Fig. 5a). We firstly analyzed their responsive patterns 0,1 , and $3 \mathrm{~d}$ after soil-drenched treatment with $0.5 \mathrm{mmol} / \mathrm{L}$ PBZ. The results demonstrated that both of the elements responded to PBZ induction (Figs. 5b and 5c). We then became interested in whether they were also responsive to SA. Intriguingly, while GST1 element still showed a response to SA, the HSRE element did not show a significant response (Figs. $5 \mathrm{~b}$ and $5 \mathrm{c}$ ). This indicates that HSRE is likely responsive to the signal(s) in the PBZ-triggered signal pathway(s) upstream of SA accumulation. This finding suggests that the HSRE element can be exploited as an ideal responsive marker to further analyze the early signal transduction triggered by PBZ.

3.5 Both $\mathrm{Ca}^{2+}$ influx and kinase activity are involved in the early signal transduction triggered by PBZ

The $\mathrm{Ca}^{2+}$ signal and kinase activity as well as reactive oxygen intermediates (ROIs) play important roles in plant HR (Alvarez et al., 1998; Torres et al., 2006; Ma and Berkowitz, 2007). To determine the 
relationship between $\mathrm{Ca}^{2+}$ influx and kinase activity and response of the HSRE element to PBZ induction, we performed analyses by using a calcium signaling blocker and kinase activity inhibitors. Pre-treatment of Arabidopsis leaves with the calcium channel blocker lanthanum III acetylacetonate hydrate $\left(\mathrm{La}^{3+}\right)$ totally suppressed the response of the HSRE to PBZ induction. In addition, treatments with a broad-range protein kinase antagonist, K252a, and a specific mitogen-activated protein kinase (MAPK) inhibitor, apigenin, also abolished the responsiveness of the HSRE elements to PBZ induction (Fig. 6). These results collectively suggest that both $\mathrm{Ca}^{2+}$ influx and kinase activity, especially MAPK activity, were involved in the early signal transduction triggered by PBZ.

(a)

GST1: TTCTAGCCACCAGATTTGACCAAAC HSRE: AACTCCAAAATTTTGTAAAATTCTTTGA

(b)

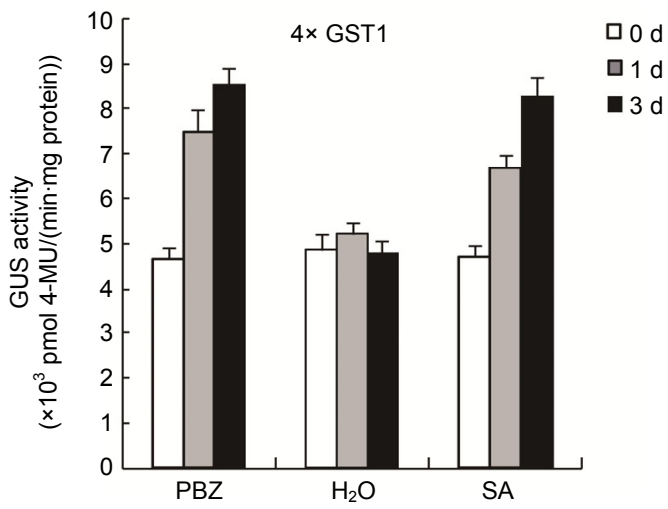

(c)

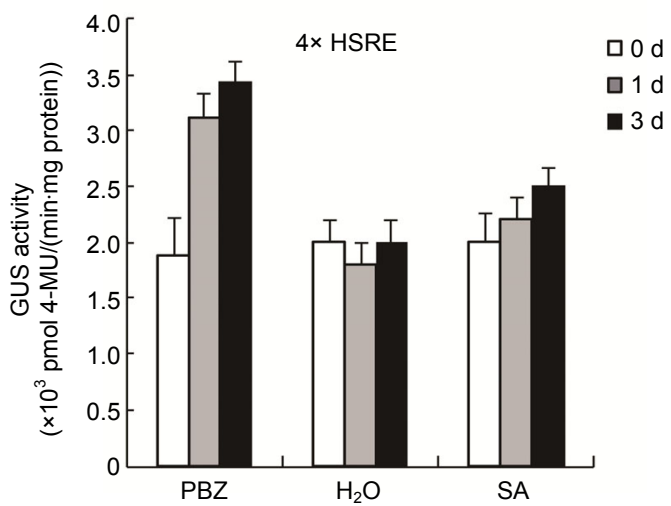

Fig. 5 Responsiveness of GST1 and HSRE elements to PBZ and SA

(a) Sequences of the GST1 and HSRE elements. (b) GUS activity in the leaves of $4 \times$ GST1 transgenic T3 lines. (c) GUS activity in the leaves of $4 \times$ HSRE transgenic T3 lines. 4-MU: 4-methylumbelliferone. Error bars indicate standard deviations $(n=6)$

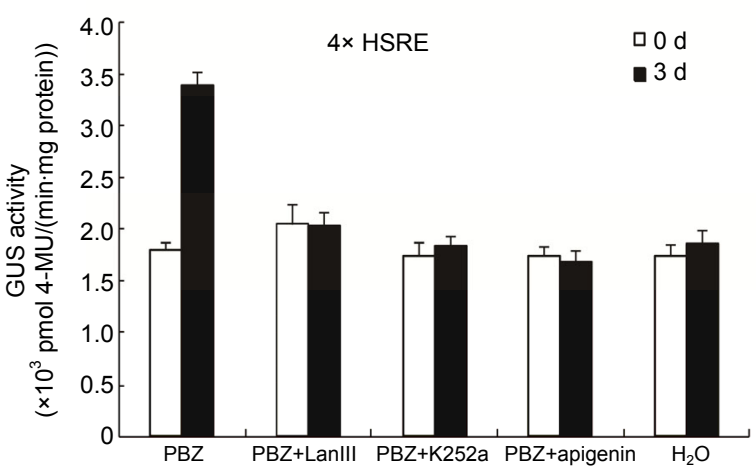

Fig. 6 Influence of calcium channel block and kinase inhibitors on responsiveness of HSRE elements to PBZ $\mathrm{La}^{3+}, \mathrm{K} 252 \mathrm{a}$, and apigenin were used to pretreat Arabidopsis leaves before $0.5 \mathrm{mmol} / \mathrm{L} \mathrm{PBZ}$ or water treatment. 4-MU: 4-methylumbelliferone. Error bars indicate standard deviations $(n=6)$

\subsection{WRKY protein-binding box is capable of re- sponding to $\mathrm{PBZ}$ induction in Arabidopsis}

Extensive studies have shown that WRKY proteins have regulatory functions in plant immune response (Rushton et al., 2010). Our previous study showed that two of their three binding sites, W-box (TTGAC), in the promoter of AtNPRI are essential for the full responsiveness of AtNPR1 to PBZ treatment (Yu et al., 2010a). Moreover, microarray analysis showed that about 15 WRKY family transcription factors were induced in Col- 0 after PBZ treatment $(\mathrm{Yu}$ et al., 2010a). To study the PBZ-triggered signal transduction in SAR, we further examined the PBZ responsive gene expression in PBZ-treated NPR1overexpressing transgenic plants in a new microarray experiment. More WRKY family transcription factors (39 members) were found to be up-regulated with at least a two-fold increase of their transcripts $8 \mathrm{~d}$ after PBZ treatment (Table 1). This finding strongly suggests that WRKY proteins are extensively involved in PBZ-triggered signaling pathways. To further elucidate the importance of WRKY proteins in the responsiveness to PBZ induction, we generated an inducible construct with tandem repeats of two W-boxes $(2 \times$ W-box) and subsequently analyzed its transgenic lines (Fig. 7a). Although a slight background in GUS staining was detected in their leaves (Fig. 7b), a significant responsiveness in both $G U S$ gene transcription and GUS activity to PBZ treatment was observed in the stable transgenic lines (Figs. 7c and 7d). These observations indicate that $\mathrm{W}$-box(es) alone is capable of responding to $\mathrm{PBZ}$ induction. 
Table 1 PBZ-induced WRKY genes in NPR1-overexpressing Arabidopsis

\begin{tabular}{|c|c|c|}
\hline Gene ID & Annotation & $\begin{array}{l}\text { Fold change }\left(\log _{2}\right) \\
\text { PBZ-8 d vs. } \mathrm{H}_{2} \mathrm{O}-8 \mathrm{~d}\end{array}$ \\
\hline AT5G56270 & WRKY2 & 1.0408 \\
\hline AT1G62300 & WRKYG & 4.4347 \\
\hline AT5G46350 & WRKY8 & 3.2967 \\
\hline AT4G39410 & WRKY13 & 1.4983 \\
\hline AT1G30650 & WRKY14 & 1.3503 \\
\hline AT2G23320 & WRKY 15 & 2.4708 \\
\hline AT2G24570 & WRKY17 & 1.9240 \\
\hline AT4G31800 & WRKY18 & 3.4418 \\
\hline AT4G01250 & WRKY 22 & 1.5641 \\
\hline AT2G30250 & WRKY25 & 2.7352 \\
\hline AT5G07100 & WRKY26 & 5.0472 \\
\hline AT5G52830 & WRKY27 & 2.4086 \\
\hline AT5G24110 & WRKY30 & 5.9014 \\
\hline AT4G22070 & WRKY31 & 3.3596 \\
\hline AT2G38470 & WRKY33 & 1.1953 \\
\hline AT1G69810 & WRKY36 & 3.5674 \\
\hline AT5G22570 & WRKY38 & 4.0561 \\
\hline AT1G80840 & WRKY40 & 1.7052 \\
\hline AT4G11070 & WRKY41 & 6.1246 \\
\hline AT3G01970 & WRKY45 & 2.1999 \\
\hline AT2G46400 & WRKY46 & 3.8088 \\
\hline AT4G01720 & WRKY47 & 4.1209 \\
\hline AT5G49520 & WRKY48 & 3.8653 \\
\hline AT5G26170 & WRKY50 & 4.7801 \\
\hline AT5G64810 & WRKY51 & 5.0924 \\
\hline AT4G23810 & WRKY53 & 4.6245 \\
\hline AT2G40740 & WRKY55 & 5.0885 \\
\hline AT1G69310 & WRKY57 & 1.3964 \\
\hline AT3G01080 & WRKY 58 & 1.4476 \\
\hline AT2G21900 & WRKY59 & 2.4599 \\
\hline AT2G25000 & WRKY60 & 3.9039 \\
\hline AT1G18860 & WRKY61 & 3.1380 \\
\hline AT5G01900 & WRKY62 & 4.0745 \\
\hline AT1G66600 & WRKY63 & 2.6046 \\
\hline AT1G29280 & WRKY 65 & 4.1438 \\
\hline AT1G80590 & WRKY66 & 2.3205 \\
\hline AT1G29860 & WRKY71 & 2.1442 \\
\hline AT5G15130 & WRKY72 & 2.4972 \\
\hline AT5G13080 & WRKY75 & 9.1676 \\
\hline
\end{tabular}

A list of PBZ-induced $W R K Y$ genes that were identified in the microarray experiment. RNA samples from NPR1-overexpressing Arabidopsis plants treated with PBZ for $8 \mathrm{~d}$ were compared with RNA samples from NPRI-overexpressing Arabidopsis plants treated with water for $8 \mathrm{~d}$. Note that the fold change values are $\log$ base 2 transformed. The twofold difference in the expression level between the PBZ- $8 \mathrm{~d}$ and $\mathrm{H}_{2} \mathrm{O}-8 \mathrm{~d}$ samples was set as the threshold for considering a gene to be PBZ-inducible
3.7 A synthetic promoter containing combinations of different cis-elements for PBZ-inducible system

To develop an effective strategy for creating inducible promoters with high responsiveness to PBZ induction, we attempted to combine distinctive elements, which have been previously shown to significantly respond to PBZ induction, including GST1, W-box, and HSRE. An inducible construct was generated with $2 \times$ GST1, 6× W-box, and $2 \times$ HSRE (named as GWH) (Fig. 8a) and its transgenic plants were then analyzed. A significantly higher GUS activity was detected in the leaves of GWH transgenic lines than in those of transgenic plants carrying single W-box or HSRE cis-elements after $4 \mathrm{~d}$ PBZ treatment (Figs. $8 \mathrm{~b}$ and $8 \mathrm{c}$ ). This result suggests that a more responsive synthetic promoter can be constructed by selectively combining distinctive $c i s$-elements.

\section{Discussion}

In this study, by using the efficient stable expression system of Arabidopsis, we clearly demonstrated that a list of individual pathogen-responsive cis-acting elements is capable of responding to PBZ induction, suggesting that the respective transcription factors are sufficient to confer a responsiveness to PBZ. These results thus revealed important regulatory nodes on the signal transduction pathways triggered by PBZ, which may in turn help to further uncover the underlying mechanism of PBZ-induced immune response. First, we provided direct evidence that both JA and ethylene signaling pathways are likely involved in the PBZ-induced SAR. An early study showed that, while PBZ induced a resistance in rice at 4-leaf stage to $M$. grisea, no SA accumulation was observed during this process. Moreover, exogenously applying SA had no detectable effect in terms of the M. grisea resistance (Iwai et al., 2007). These data indicate that the immune response triggered by PBZ in rice is not likely related to the accumulation of SA and some other mechanisms may be responsible for the process. This is consistent with our observations that JERE, GCC, and HSRE all respond to PBZ, indicating that, besides SA, JA and ethylene may also play important roles in PBZ-induced systemic immune responses in plants. These data collectively suggest that multiple hormone signaling pathways 
(a) $2 \times W$-box TCTAGATTATTCAGCCATCAAAGTTGACCAATAATACTAGATTATTCAGCCATCAAAGTTGACCAATAATACTAGTAAGCTTCTGCAG

(b)
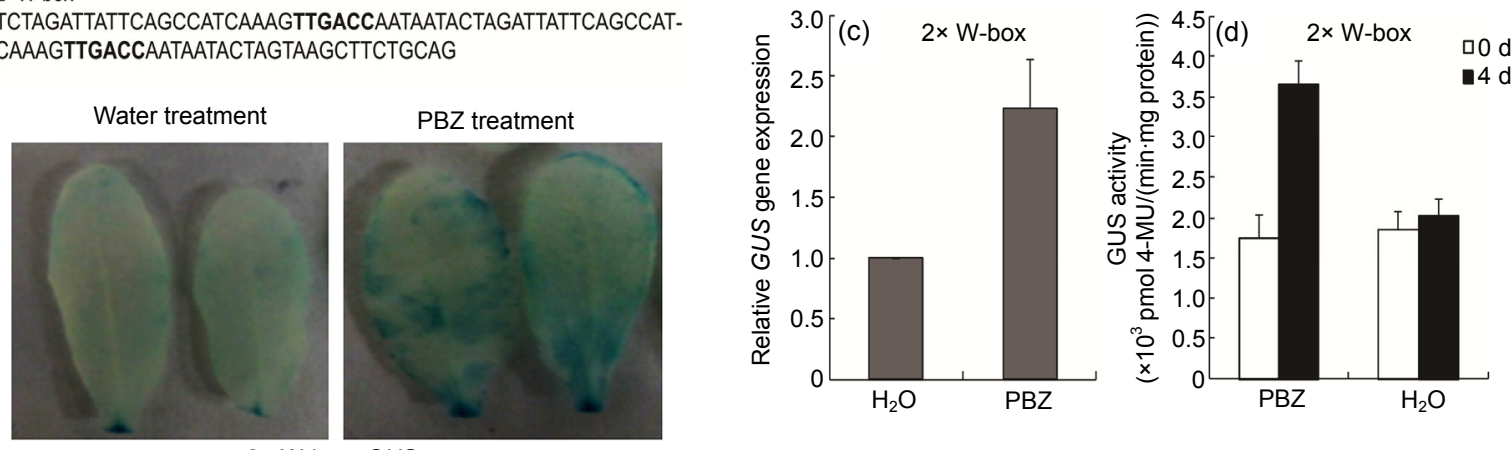

2× W-box: GUS

Fig. 7 Responsiveness of W-box elements to PBZ induction

(a) Sequence of the $2 \times \mathrm{W}$-box. Core sequences are shown in boldface. (b, c) GUS gene expression in the leaves of $2 \times \mathrm{W}$-box transgenic T3 lines $4 \mathrm{~d}$ after treatment with PBZ or water. GUS staining was used to detect the expression. The transcript levels were quantified by qRT-PCR with ACTIN2 (ACT2) as an internal control. Error bars indicate standard deviations $(n=4)$. (d) GUS activity in the leaves of $2 \times$ W-box transgenic T3 lines. 4-MU: 4-methylumbelliferone. Error bars indicate standard deviations $(n=6)$

(a) GWH

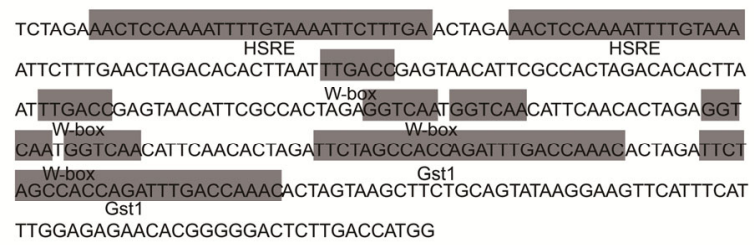

(b)

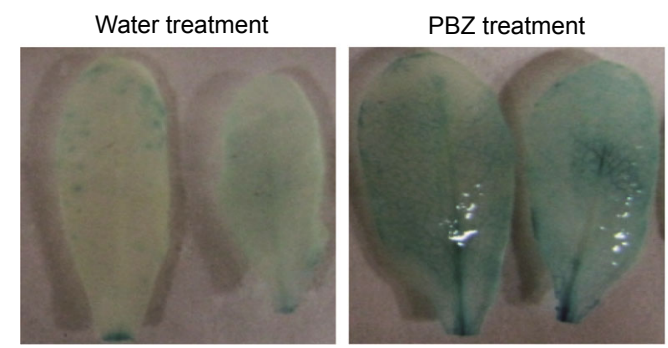

(c)

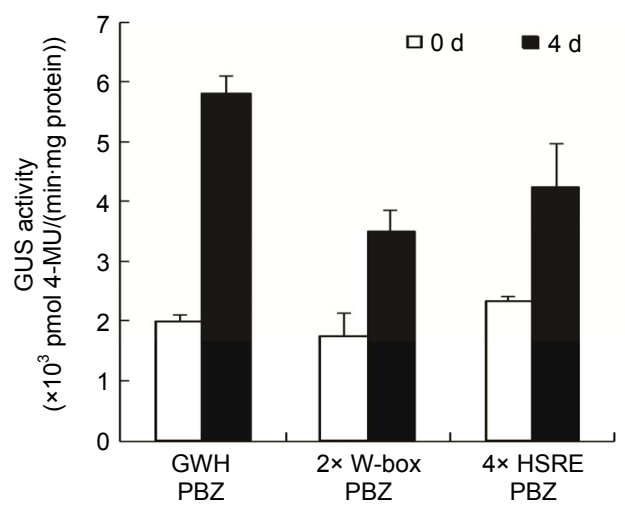

GWH: GUS

Fig. 8 Responsiveness of the synthetic promoter combining HSRE, W-box(es), and GST1 to PBZ

(a) Sequence of the synthetic promoter. The elements are shown in gray shadow. (b) GUS gene expression in the leaves of GWH transgenic T3 lines $4 \mathrm{~d}$ after treatment with PBZ or water. GUS staining was used to detect the expression. (c) GUS activity in the leaves of GWH, $2 \times$ W-box, and 4× HSRE transgenic T3 lines. 4-MU: 4-methylumbelliferone. Error bars indicate standard deviations $(n=6)$

may act together in the immune response triggered by PBZ and a complex mechanism is responsible for the regulation of cross-talks among these distinct signaling pathways.

Increasing evidence also supports that both $\mathrm{Ca}^{2+}$ elevation and kinase activity play important roles in response to both pathogen-associated molecular pattern (PAMP)-triggered immunity (PTI) and effectortriggered immunity (ETI)-associated HR (HammondKosack and Parker, 2003; Ma and Berkowitz, 2007). In addition, MAPK signaling cascades downstream of
$\mathrm{Ca}^{2+}$ elevation activate the expression of genes related to pathogen defense, including some HR-related ones (Asai et al., 2002; Zhang et al., 2006). Although it remains unclear how MAPK cascades are activated by cytosolic $\mathrm{Ca}^{2+}$ elevation, the initial phosphorylation in $\mathrm{a} \mathrm{Ca}^{2+}$-dependent manner indicates a role of calcium-dependent protein kinases (CDPKs) in such a process. With HSRE, a cis-acting element specifically responsive to $\mathrm{HR}$, we further explored the relationship between HR and PBZ-triggered immune response. We observed that pretreatment with a calcium 
channel blocker $\mathrm{La}^{3+}$, a broad-range protein kinase antagonist K252a or a MAPK-specific inhibitor apigenin abolished the effect of PBZ induction. Interestingly, in other work, we also observed that many CDPK and MAPK family members were up-regulated after PBZ treatment. Moreover, up-regulations of some earliest activated genes following pathogenassociated molecular pattern PAMP perception or $R$ gene interactions, such as EDS1, PAD4 and SAG101, have also been observed (data not shown). Based on these analyses, we hypothesize that the immune response activated by PBZ is similar to that induced by PAMP perception or $R$ gene interactions, in contrast to that triggered by other widely used plant immune response activators (i.e. benzothiadiazole (BTH), 2,6dichloroisonicotinic acid (INA), and $\beta$-aminobutyric acid (BABA)) (Serrano and Guzman, 2004; Ton and Mauch-Mani, 2004). Given the above findings, the PBZ-inducible expression system may be exploited to further explore the cross-talks among SA, JA, and ethylene signal transduction pathways, and to reveal the precise roles of $\mathrm{Ca}^{2+}$ elevation and kinase activity involved in the microbe-plant interaction and the plant systemic immune response, which would provide profound insights into the mechanisms of the plant immune response.

Pathogens of plants are a significant and growing threat to crop production worldwide. Genetic engineering has been developed as a potential approach to tackle this formidable problem (HammondKosack and Parker, 2003). However, how to properly express diversified resistance genes has been and still is a challenging problem (Gurr and Rushton, 2005). Recent progresses in the elucidation of mechanisms of pathogen-triggered immune response and chemicaltriggered immune response have made it possible to construct a novel type of transgene expression system, i.e. an immune response activator inducible transgene expression system, which can provide dual protection mechanisms, one by driving target gene expression and the other via triggering the endogenous immune system (Roslan et al., 2001; Rushton et al., 2002; Camargo et al., 2007). As a cheap, environmentally friendly, and widely used SAR activator for more than three decades, PBZ is obviously an ideal activator in this regard (Gozzo, 2003; Iwata et al., 2004). In this study, we attempted to improve promoter efficacy by combining HSRE, W-box(es), and GST1 into one synthetic promoter. A significant increase in responsiveness to PBZ was indeed observed by combining these three $c i s$-acting elements together. It is plausible for promoter efficacy to be further improved by incorporating more responsive cis-acting elements into one synthetic promoter and/or by optimizing the combinations of these elements.

\section{Acknowledgements}

We would like to thank Dr. Imre E. SMOSSICH (Max Planck Institute, Germany) for the gift of transgenic Arabidopsis seeds of GCC and JERE elements.

\section{Compliance with ethics guidelines}

Zheng ZHU, Jiong GAO, Jin-xiao YANG, Xiao-yan WANG, Guo-dong REN, Yu-long DING, and Ben-ke KUAI declare that they have no conflict of interest.

This article does not contain any studies with human or animal subjects performed by any of the authors.

\section{References}

Alvarez, M.E., Pennell, R.I., Meijer, P.J., et al., 1998. Reactive oxygen intermediates mediate a systemic signal network in the establishment of plant immunity. Cell, 92(6): 773-784. [doi:10.1016/S0092-8674(00)81405-1]

Asai, T., Tena, G., Plotnikova, J., et al., 2002. MAP kinase signalling cascade in Arabidopsis innate immunity. Nature, 415(6875):977-983. [doi:10.1038/415977a]

Camargo, A., Llamas, A., Schnell, R.A., et al., 2007. Nitrate signaling by the regulatory gene NIT2 in Chlamydomonas. Plant Cell, 19(11):3491-3503. [doi:10.1105/tpc.106.04 5922]

Clough, S.J., Bent, A.F., 1998. Floral dip: a simplified method for Agrobacterium-mediated transformation of Arabidopsis thaliana. Plant J., 16(6):735-743. [doi:10.1046/j. 1365-313x.1998.00343.x]

Gozzo, F., 2003. Systemic acquired resistance in crop protection: from nature to a chemical approach. J. Agric. Food Chem., 51(16):4487-4503. [doi:10.1021/jf030025s]

Gurr, S.J., Rushton, P.J., 2005. Engineering plants with increased disease resistance: how are we going to express it? Trends Biotechnol., 23(6):283-290. [doi:10.1016/j.tibtech. 2005.04.009]

Hammond-Kosack, K.E., Parker, J.E., 2003. Deciphering plant-pathogen communication: fresh perspectives for molecular resistance breeding. Curr. Opin. Biotechnol., 14(2):177-193. [doi:10.1016/S0958-1669(03)00035-1]

Heath, M.C., 2000. Hypersensitive response-related death. Plant Mol. Biol., 44(3):321-334. [doi:10.1023/A:1026 592509060]

Iwai, T., Seo, S., Mitsuhara, I., et al., 2007. Probenazoleinduced accumulation of salicylic acid confers resistance to Magnaporthe grisea in adult rice plants. Plant Cell Physiol., 48(7):915-924. [doi:10.1093/pcp/pcm062] 
Iwata, M., Umemura, K., Midoh, N., 2004. Probenazole $\left(\right.$ Oryzemate $\left.^{\circledR}\right)$ : a plant defense activator. In: Kawasaki, S. (Ed.), Rice Blast: Interaction with Rice and Control: Proceedings of the 3rd International Rice Blast Conference. Springer Netherlands, p.163-171. [doi:10. 1007/978-0-306-48582-4_19]

Jefferson, R.A., Kavanagh, T.A., Bevan, M.W., 1987. GUS fusions: $\beta$-glucuronidase as a sensitive and versatile gene fusion marker in higher-plants. $E M B O \quad J$, 6(13): 3901-3907.

Kirsch, C., Takamiya-Wik, M., Schmelzer, E., et al., 2000. A novel regulatory element involved in rapid activation of parsley ELI7 gene family members by fungal elicitor or pathogen infection. Mol. Plant Pathol., 1(4):243-251. [doi:10.1046/j.1364-3703.2000.00029.x]

Levine, A., Tenhaken, R., Dixon, R., et al., 1994. $\mathrm{H}_{2} \mathrm{O}_{2}$ from the oxidative burst orchestrates the plant hypersensitive disease resistance response. Cell, 79(4):583-593. [doi:10. 1016/0092-8674(94)90544-4]

Ma, W., Berkowitz, G.A., 2007. The grateful dead: calcium and cell death in plant innate immunity. Cell. Microbiol., 9(11):2571-2585. [doi:10.1111/j.1462-5822.2007.01031.x]

Mehrotra, R., Gupta, G., Sethi, R., et al., 2011. Designer promoter: an artwork of cis engineering. Plant Mol. Biol., 75(6):527-536. [doi:10.1007/s11103-011-9755-3]

Menke, F.L.H., Champion, A., Kijne, J.W., et al., 1999. A novel jasmonate- and elicitor-responsive element in the periwinkle secondary metabolite biosynthetic gene Str interacts with a jasmonate- and elicitor-inducible AP2domain transcription factor, ORCA2. EMBO J., 18(16): 4455-4463. [doi:10.1093/emboj/18.16.4455]

Midoh, N., Iwata, M., 1996. Cloning and characterization of a probenazole-inducible gene for an intracellular pathogenesis-related protein in rice. Plant Cell Physiol., 37(1):9-18. [doi:10.1093/oxfordjournals.pcp.a028918]

Midoh, N., Iwata, M., 1997. Expression of defense-related genes by probenazole or 1,2-benzisothiazole-3(2H)-one 1,1-dioxide. J. Pesticide Sci., 22(1):45-47. [doi:10.1584/ jpestics.22.45]

Nakashita, H., Yoshioka, K., Yasuda, M., et al., 2002. Probenazole induces systemic acquired resistance in tobacco through salicylic acid accumulation. Physiol. Mol. Plant Pathol., 61(4):197-203. [doi:10.1006/pmpp.2002.0426]

Nemhauser, J.L., Hong, F.X., Chory, J., 2006. Different plant hormones regulate similar processes through largely nonoverlapping transcriptional responses. Cell, 126(3): 467-475. [doi:10.1016/j.cell.2006.05.050]

Nikolaev, O.N., Aver'yanov, A.A., 1991. Involvement of superoxide radical in the mechanism of fungicidic action of phthalide and probenazol. Soviet Plant Physiol., 38(3): 375-381.

Ohme-Takagi, M., Suzuki, K., Shinshi, H., 2000. Regulation of ethylene-induced transcription of defense genes. Plant Cell Physiol., 41(11):1187-1192. [doi:10.1093/pcp/pcd057]

Penninckx, I.A.M.A., Eggermont, K., Terras, F.R.G., et al., 1996. Pathogen-induced systemic activation of a plant defensin gene in Arabidopsis follows a salicylic acidindependent pathway. Plant Cell, 8(12):2309-2323. [doi:10.2307/3870470]

Pontier, D., Balague, C., Bezombes-Marion, I., et al., 2001. Identification of a novel pathogen-responsive element in the promoter of the tobacco gene HSR203J, a molecular marker of the hypersensitive response. Plant J., 26(5): 495-507. [doi:10.1046/j.1365-313x.2001.01049.x]

Roslan, H.A., Salter, M.G., Wood, C.D., et al., 2001. Characterization of the ethanol-inducible alc gene-expression system in Arabidopsis thaliana. Plant J., 28(2):225-235. [doi:10.1046/j.1365-313X.2001.01146.x]

Rushton, P.J., Somssich, I.E., 1998. Transcriptional control of plant genes responsive to pathogens. Curr. Opin. Plant Biol., 1(4):311-315. [doi:10.1016/1369-5266(88)80052-9]

Rushton, P.J., Reinstadler, A., Lipka, V., et al., 2002. Synthetic plant promoters containing defined regulatory elements provide novel insights into pathogen- and wound-induced signaling. Plant Cell, 14(4):749-762. [doi:10.1105/tpc. 010412]

Rushton, P.J., Somssich, I.E., Ringler, P., et al., 2010. WRKY transcription factors. Trends Plant Sci., 15(5):247-258. [doi:10.1016/j.tplants.2010.02.006]

Sakamoto, K., Tada, Y., Yokozeki, Y., et al., 1999. Chemical induction of disease resistance in rice is correlated with the expression of a gene encoding a nucleotide binding site and leucine-rich repeats. Plant Mol. Biol., 40(5): 847-855. [doi:10.1023/A:1006244323934]

Sasaki, K., Iwai, T., Hiraga, S., et al., 2004. Ten rice peroxidases redundantly respond to multiple stresses including infection with rice blast fungus. Plant Cell Physiol., 45(10):1442-1452. [doi:10.1093/pcp/pch165]

Sekizawa, Y., Watanabe, T., 1981. On the mode of action of probenazole against rice blast. J. Pesticide Sci., 6(2): 247-255. [doi:10.1584/jpestics.6.247]

Serrano, M., Guzman, P., 2004. Isolation and gene expression analysis of Arabidopsis thaliana mutants with constitutive expression of $A T L 2$, an early elicitor-response RING-H2 zinc-finger gene. Genetics, 167(2):919-929. [doi:10.1534/ genetics.104.028043]

Shah, J., Klessig, D.F., 1996. Identification of a salicylic acid-responsive element in the promoter of the tobacco pathogenesis-related $\beta$-1,3-glucanase gene, $P R$ - $2 d$. Plant J., 10(6):1089-1101. [doi:10.1046/j.1365-313X.1996. 10061089.x]

Strittmatter, G., Gheysen, G., Gianinazzipearson, V., et al., 1996. Infections with various types of organisms stimulate transcription from a short promoter fragment of the potato gst1 gene. Mol. Plant-Microbe Interact., 9(1): 68-73. [doi:10.1094/MPMI-9-0068]

Ton, J., Mauch-Mani, B., 2004. $\beta$-amino-butyric acid-induced resistance against necrotrophic pathogens is based on ABA-dependent priming for callose. Plant J., 38(1): 119-130. [doi:10.1111/j.1365-313X.2004.02028.x]

Torres, M.A., Jones, J.D., Dangl, J.L., 2006. Reactive oxygen species signaling in response to pathogens. Plant Physiol., 
141(2):373-378. [doi:10.1104/pp.106.079467]

Venter, M., 2007. Synthetic promoters: genetic control through cis engineering. Trends Plant Sci., 12(3):118-124. [doi:10.1016/j.tplants.2007.01.002]

Watanabe, T., 1977. Effects of probenazole $\left(\right.$ Oryzemate $\left.{ }^{\circledR}\right)$ on each stage of rice blast fungus (Pyricularia oryzae Cavara) in its life cycle. J. Pesticide Sci., 2(4):395-404. [doi:10. 1584/jpestics.2.395]

Yasuda, M., Ishikawa, A., Jikumaru, Y., et al., 2008. Antagonistic interaction between systemic acquired resistance and the abscisic acid-mediated abiotic stress response in Arabidopsis. Plant Cell, 20(6):1678-1692. [doi:10.1105/tpc.107.054296]

Yoshioka, K., Nakashita, H., Klessig, D.F., et al., 2001. Probenazole induces systemic acquired resistance in Arabidopsis with a novel type of action. Plant J., 25(2): 149-157. [doi:10.1046/j.1365-313x.2001.00952.x]

Yu, J., Wang, X.Y., Wei, Q.A., et al., 2010a. Identification of regulatory cis-elements upstream of AtNPRI that are responsive to probenazole treatment in transgenic tobacco plants. J. Plant Biol., 53(4):282-290. [doi:10.1007/ s12374-010-9115-x]

Yu, J., Gao, J.O., Wang, X.Y., et al., 2010b. The pathway and regulation of salicylic acid biosynthesis in probenazoletreated Arabidopsis. J. Plant Biol., 53(6):417-424. [doi:10.1007/s12374-010-9130-y]

Zhang, A.Y., Jiang, M.Y., Zhang, J.H., et al., 2006. Mitogenactivated protein kinase is involved in abscisic acidinduced antioxidant defense and acts downstream of reactive oxygen species production in leaves of maize plants. Plant Physiol., 141(2):475-487. [doi:10.1104/pp. 105.075416]

\section{List of electronic supplementary materials}

Table S1 Expression level comparison of the shared genes that are up-regulated by both PBZ and MeJA in Arabidopsis

Table S2 Expression level comparison of the shared genes that are up-regulated by both PBZ and ACC in Arabidopsis

\section{中文概要}

题 目: 基于已知顺式元件的人工启动子响应性分析揭示 烯丙异噻唑诱导系统可触发植物体内多条信号 途径

目 的：构建有效响应烯丙异噻唑 (PBZ) 诱导的人工合 成启动子, 了解植物体内受 PBZ 诱导系统触发的 信号途径。

创新点: 通过分析包含已知顺式元件的人工合成启动子对 PBZ 的响应性, 为构建一种基于 PBZ 诱导系统的 新型化学诱导启动子提供了可能性, 并初步揭示 了除水杨酸 $(\mathrm{SA})$ 外, 茉莉酸 (JA) 和乙烯等多 条信号途径可能共同参与了 PBZ 诱导的植物免 疫反应过程。

方 法: 利用已知的响应相关信号通路的顺式作用元件构 建人工合成启动子, 融合 $G U S$ 报告基因后, 稳定 转化拟南芥。通过检测 PBZ 处理过程中 GUS 酶 活性的变化, 了解人工合成启动子对 PBZ 的响应 性, 分析 PBZ 诱导系统可能触发的信号途径。

结 论: 除了 SA 响应元件 SARE 可以有效响应 PBZ 诱导 外, 利用 JA 和乙烯响应元件 JERE 和 GCC, 超 敏反应（HR）相关的顺式元件 HSRE 和 GST1, 以及植物抗病反应中重要顺式作用元件 W-box 构建的人工合成启动子也均可有效响应 PBZ。另 外, 通过人工合成启动子响应性分析的手段, 初 步揭示了包括 SA、JA、乙烯、钙离子和丝裂原 活化蛋白激酶 (MAPKs) 在内的多条信号通路可 能共同参与了 $\mathrm{PBZ}$ 诱导植物免疫反应的过程。

关键词: 烯丙异噻唑; 人工合成启动子; 顺式作用元件; 化学诱导系统 